\title{
A UNIFIED QUADRATIC SEMI-INFINITE PROGRAMMING APPROACH TO TIME AND FREQUENCY DOMAIN CONSTRAINED DIGITAL FILTER DESIGN*
}

\author{
Y. $\mathrm{LIU}^{\dagger}$, C. H. TSENG ${ }^{\ddagger}$, AND K. L. TEO
}

\begin{abstract}
A unified quadratic semi-infinite programming approach is introduced to solve digital filter design problems with time or frequency-domain specification. An algorithm based on this approach is developed and the corresponding convergence result is presented. This computational method is then applied to the optimum filter design problems subject to time and frequency domain specifications, namely the time domain envelope constrained filter design problems and the frequencydomain least square FIR filter design problems. For illustration, two examples are given.
\end{abstract}

1. Introduction. In signal processing, many filter design problems can often be cast as constrained optimization problems where the constraints are defined by the specifications of the filter or by the requirements on the output signal. These constraints can arise either from practical considerations or from the standards set by certain regulatory bodies(see e.g., [4]).

In particular, some constraints required to be satisfied are continuous constraints in either time or frequency domain. Examples include (i) the optimum envelopeconstrained filter design problem $[16,17,18]$, where the response of the filter corresponding to a given excitation is required to stay within a specified tolerance about the desired response in the time domain; and (ii) the optimal least square FIR filtering problem subject to maximum error constraints in frequency domain [5]. These optimum filter design problems can be formulated as appropriate quadratic semi-infinite programming problems. Many approaches have been proposed to deal with this class of optimization problems. The simplest one is to approximate the problem as a finite quadratic optimization problem via discretization of the index set of the infinite constraints. However, there is no guarantee that the continuous constraints will be satisfied in between the discretization points. However, many methods, including the quadratic programming methods, the $H_{\infty}$ method ([6],[11],[17]), and the $H_{2}$ methods ([10]), have been used to solve this discretised quadratic programming problem under various scenarios. The constraint transcription techniques proposed in [12] and [13]

*Received on September 10, 2002; accepted for publication on october 29, 2002. This paper is dedicated to Professor John B. Moore on the occasion of his 60th birthday. This research was supported by a research grant (Polyu 5101/01E) from the Research Grant Council of Hong Kong.

${ }^{\dagger}$ Department of Applied Mathematics, The Hong Kong Polytechnic University, Hung Hom, Kowloon, Hong Kong. E-mail: mayliu@inet.polyu.edu.hk,

${ }^{\ddagger}$ School of Engineering, University of Warwick, Coventry CV4 7AL, UK.,

${ }^{\S}$ Department of Applied Mathematics, and Centre for Multimedia Singal Processing, Department of Electric and Information Engineering, The Hong Kong Polytechnic University, Hung Hom, Kowloon, Hong Kong 
are popular choices for dealing with semi-infinite programming problems (see [16]). However, these methods require numerical integration over the index set and feasibility check of the continuous constraint and hence are numerically expensive. Recently, a new algorithm based on the dual parameterization approach is developed in [8] for solving quadratic semi-infinite programming problems. The convergence of the algorithm is proved.

In this paper, the theory and algorithm obtained in [8] are extended to quadratic semi-infinite programming problems involving multiple continuous constraints. These results are then shown to give rise to a unified approach to the optimum filter design problems with time and frequency domain specifications.

The rest of the paper is organized as follows: In Section 2, a general quadratic semi-infinite programming problem involving several continuous inequality constraints formulated and the dual parameterization technique proposed in [7] is used to develop a computational algorithm. Convergence result is given. In Sections 3 and 4, numerical examples of both time and frequency domain filter design problems are solved by using the proposed algorithm. Finally, Section 5 concludes the paper.

2. Quadratic Semi-Infinite Programming Algorithm. In this section, we present an algorithm for solving the following class of quadratic semi-infinite programming problems which covers the constrained optimal filter design problems with frequency and time domain specifications considered in [5] and [15], respectively.

Problem $(\mathrm{P})$

$$
\begin{gathered}
\min _{x} f(x) \hat{=} \frac{1}{2} x^{\prime} Q x+c^{\prime} x \\
\text { subject to } g(x, \tau) \hat{=} A(\tau) x-b(\tau) \leq 0, \text { for all } \tau \in \Omega,
\end{gathered}
$$

where the index set $\Omega$ is a compact subset of $R^{s}, Q \in R^{N \times N}$ is a positive definite matrix, $c \in R^{N}, A(\cdot) \in C\left(\Omega, R^{m \times N}\right)$ and $b(\cdot) \in C\left(\Omega, R^{m}\right)$, while $C\left(\Omega, R^{m \times N}\right)$ and $C\left(\Omega, R^{m}\right)$ denote, respectively, the classical Banach spaces consisting of all continuous functions defined on $\Omega$ with value in $R^{m \times N}$ and $R^{m}$. For numerical reasons, we further assume that $A(\cdot)$ and $b(\cdot)$ are continuously differentiable on $\Omega$.

Through out this paper, we assume that the Slater constraint qualification holds at some point $x^{0} \in R^{N}$, i.e., $g\left(x^{0}, \tau\right)<0_{m}$ for all $\tau \in \Omega$.

Let $A: R^{N} \rightarrow C\left(\Omega, R^{m}\right)$ be the operator defined by the matrix function $A(\cdot)$ according to

$$
(A x)(\tau)=A(\tau) x \text { for } \tau \in \Omega
$$

and denote by $A^{*}$ the dual operator of $A$. Using the above notations, problem (P) can be stated as

$$
\begin{aligned}
& \min _{x}(1 / 2) x^{\prime} Q x+c^{\prime} x, \\
& \text { s.t. } A x-b \leq 0 .
\end{aligned}
$$


The Dorn's dual of problem $(\mathrm{P})$ can be written as:

Problem (D)

$$
\begin{array}{cl}
\min _{x, \Lambda} & L(x, \Lambda) \\
\text { s.t. } & Q x+c+A^{*} \Lambda=0 \\
& \Lambda \geq 0
\end{array}
$$

where

$$
L(x, \Lambda)=(1 / 2) x^{\prime} Q x+\int_{Y} b(y) d \Lambda(y) .
$$

We state the well known KKT conditions for problem (P) as the following theorem.

TheOREM 2.1 (KKT conditions). Let the Slater constraint qualification be satisfied. The minimum of problem $(P)$ is achieved at $x^{*} \in R^{n}$ if and only if $x^{*}$ is feasible and there exists a $\Lambda^{*} \in M(\Omega)$ such that

$$
\begin{aligned}
& Q x^{*}+b+A^{*} \Lambda^{*}=0, \\
& \int_{\Omega}\left(A(\tau) x^{*}-b(\tau)\right) d \Lambda^{*}(\tau)=0, \\
& \Lambda^{*} \geq 0 .
\end{aligned}
$$

The result of [7] can be easily extended to the class of problems described by problem (P) as follows. The proof is similar to that of [7] and hence is omitted.

Theorem 2.2. Let the Slater constraint qualification be satisfied. Assume that the optimal solution of the primal problem $(P)$ is achieved at $x^{*} \in R^{N}$. Then, the set of multipliers satisfying the KKT conditions of problem $(P)$ necessarily includes a measure with finite support at no more than $N$ points.

We note, by Theorem 2.2, that there exits a solution pair $\left(x^{*}, \Lambda^{*}\right)$ of the dual problem (D) of which the measure $\Lambda^{*}$ has a finite support of no more than $N$ points. Thus, according to the argument of [7], the dual semi-infinite problem (D) can be reduced to the following finite dimensional optimization problem (PD), called the parameterized dual of problem $(\mathrm{P})$.

$$
\begin{aligned}
& \min _{(x, t, \lambda)} L_{k}(x, t, \lambda) \\
& \text { s.t. } \lambda_{i} \geq 0, \tau_{i} \in \Omega, i=1,2, \cdots, k
\end{aligned}
$$

where the integer $k$ is the parameterization number, $t=\left(\tau_{1}, \ldots, \tau_{k}\right), \lambda=\left[\lambda_{1} \lambda_{2} \ldots\right.$ $\left.\lambda_{k}\right], \lambda_{i}=\left[\begin{array}{llll}\lambda_{i, 1} & \lambda_{i, 2} & \cdots & \lambda_{i, m}\end{array}\right]^{\prime} \in R_{m}$ for $i=1,2, \cdots, k$, and the cost function $L_{k}(x, t, \lambda)$ is given by

$$
L_{k}(x, t, \lambda)=\frac{1}{2} \mathbf{x}^{\prime} Q x+\sum_{i=1}^{k} b^{\prime}\left(\tau_{i}\right) \lambda_{i} .
$$

According to the dual parameterization theory, once a solution $(x, t, \lambda)$ is obtained for problem $(\mathrm{PD})$, the optimal solution to the primal problem $(\mathrm{P})$ is $x$. To state 
the algorithm for problem $(\mathrm{P})$, we denote by $(\mathrm{PD}(t))$ the problem obtained from problem (PD) by fixing $t$. It is easy to see that $(\mathrm{PD}(t))$ is the dual problem of the following problem $(\mathrm{P}(t))$ for fixed $\tau_{i} \in \Omega, i=1,2, \cdots, k$ :

$$
\begin{array}{cl}
\min _{x} & f(x), \quad x \in R^{N} \\
\text { subject to } & g\left(x, \tau_{i}\right) \leq 0_{m}, i=1, \ldots, k .
\end{array}
$$

Theorem 2.3. ([8]) Consider problems $(P),(P(t))$ and $(P D)$. The following statements hold.

(i). Let $\bar{x}$ be an optimum solution to problem $(P(t))$. If $\bar{x}$ satisfies the infinite constraint (2.2), then it is the optimal solution for the primal problem (P).

(ii). Let $v_{k}$ be the optimal value of problem (PD) with parameterization number $k$. The sequence $\left\{v_{k}\right\}$ is decreasing, and there is an $k^{*}$ such that $v_{k^{*}}=v_{k}$, for all $k \geq k^{*}$. Furthermore, if $k^{*} \geq 1$, then $v_{k^{*}-1}>v_{k^{*}}$.

(iii). The number $k^{*}$ in (ii) is the minimum integer such that for $k \geq k^{*}$, a global solution of the finite problem (PD) provides the solution for the primal problem $(P)$ in the sense that if $\left(x^{*}, t^{*}, \lambda^{*}\right)$ is a global optimizer of problem $(P D)$, then $x^{*}$ is the global optimizer of the primal problem $(P)$.

(iv). If $0 \leq k<k^{*}$, then $v_{k}>v_{k+1}$.

Note that the number $k^{*}$ appeared in Theorem 2.3 is called the minimum parameterization number. If the optimal primal solution is an interior point of the feasible region, then $k^{*}=0$.

Let $\left\{k_{i}\right\}$ be a given sequence of the parameterization numbers satisfying $k_{i} \leq k_{i+1}$. For each $i$, let $\Omega_{i}=\left\{\tau_{j}^{i}: j=1, \ldots, k_{i}\right\}$ be a given subset of $\Omega$, and let $t^{i}=\left(\tau_{1}^{i}, \ldots, \tau_{k_{i}}^{i}\right)$. Define the density distance between $\Omega_{i}$ and $\Omega$ as:

$$
d\left(\Omega_{i}, \Omega\right) \triangleq \max _{\tau \in \Omega} \min _{1 \leq j \leq \ell_{i}}\left|\tau-\tau_{j}^{i}\right|
$$

TheOrem 2.4. [8] Let the sequence $\left\{t^{i}\right\}$ be given as above. Suppose that $\left(\bar{x}^{i}, \bar{\lambda}^{i}\right)$ is a solution of problem $\left(P D\left(t^{i}\right)\right)$. If

$$
d\left(\Omega_{i}, \Omega\right) \rightarrow 0 \text { as } i \rightarrow \infty,
$$

then it holds that

(i). $\left\{\bar{x}^{i}\right\}$ converges to the solution of the primal problem $(P)$.

(ii). $v\left(P D\left(t^{i}\right)\right) \rightarrow v(D)$, where $v(S)$ denotes the optimal value of a given problem $(S)$.

The optimization algorithm may now be stated as follows:

Algorithm 2.1.

Step 0 (Initialization) Give a small number $\epsilon>0$. Choose a sequence of index sets $\left\{\Omega_{i}\right\}$. Set $i=1$. 
Step 1 (Compute a local optimum) Solve the finite problem $\left(P D\left(t^{i}\right)\right)$. Denote the local optimal solution by $\left(x^{i}, \lambda^{i}\right)$.

Step 2 (Test improvement of the objective) If $i \geq 2$ and $\left|v\left(P D\left(t^{i}\right)\right)-v(D)\right| \leq \epsilon$, go to Step 3, else $i=i+1$ and go to Step 1.

Step 3 (Compute the global optimum) Implement a local search for the finite dual problem (PD) with $k=k_{i}$. The solution is denoted by $\left(x^{*}, t^{*}, \lambda^{*}\right)$. Then, $x^{*}$ is taken as the optimizer for problem $(P)$.

3. Frequency-Domain Least Square FIR Filter Design. Consider the design of linear-phase FIR digital filters in which the objective is to minimize the weighted least square error of the actual response with respect to a desired response, subject to maximum allowable error between the actual and the desired responses in the passband and stopband. The filter to be designed is a low-pass channel filter and the corresponding optimization problem is referred to as the peak constrained weighted least square error (PCWLSE) filter design $[1,2,5]$ problem. The actual and desired frequency responses are, respectively, given by

$$
H(x, \omega)=A(x, \omega) e^{-j \theta(\omega)}
$$

and

$$
H_{d}(\omega)=A_{d}(\omega) e^{-j \theta_{d}(\omega)}
$$

where $A(x, \omega)$ and $A_{d}(\omega)$ are real amplitudes of the frequency responses, and $\theta(\omega)$ and $\theta_{d}(\omega)$ are continuous phase responses. The frequency domain is divided into the passband $\Omega_{p}=\left[0, \omega_{p}\right]$, the transition band (or “don't care" band), and the stopband $\Omega_{s}=\left[\omega_{s}, 0.5\right]$. Furthermore, $A(x, \omega)$ and $A_{d}(\omega)$ are, respectively, given by

$$
A(x, \omega)=\xi^{\prime}(\omega) x, \quad \forall \omega \in \Omega_{p} \cup \Omega_{s}
$$

and

$$
A_{d}(\omega)=\left\{\begin{array}{ll}
1, & \omega \in \Omega_{p} \\
0, & \omega \in \Omega_{s}
\end{array} .\right.
$$

Note that the impulse response $x(\ell)=x(N-1-\ell), 0 \leq \ell \leq N$ is symmetric. Thus, the column vector $x$ contains $n$ impulse response coefficients where $n=(N+1) / 2$ if $N$ is odd and $n=N / 2$ if $N$ is even. $\xi(\omega)$ is a column vector containing cosine functions of $\omega$ (see [9]). The phase response is

$$
\theta(\omega)=2 \pi \omega(N-1) / 2,
$$

and the real amplitude corresponding to the actual frequency response is

$$
A(x, \omega)= \begin{cases}\sum_{\ell=0}^{(N-1) / 2} a(\ell) \cos (2 \pi \omega \ell) & N \text { is odd } \\ \sum_{\ell=1}^{N / 2} b(\ell) \cos [2 \pi \omega(\ell-1 / 2)] & N \text { is even }\end{cases}
$$


where $a(0)=x\left(\frac{N-1}{2}\right), a(\ell)=2 x\left(\frac{N-1}{2}-\ell\right), \ell=1, \ldots, \frac{N-1}{2}$, and $b(\ell)=2 x(N / 2-\ell), \ell=$ $1, \ldots, N / 2$. The cost function associated with the PCWLSE filter design problem is then given by

$$
\begin{aligned}
& \int_{\Omega_{p} \cup \Omega_{s}}\left\{W(\omega)\left|A(x, \omega)-A_{d}(\omega)\right|^{2}\right\} d \omega \\
= & W_{p} \int_{\Omega_{p}}\left|A(x, \omega)-A_{d}(\omega)\right|^{2} d \omega+W_{s} \int_{\Omega_{s}}\left|A(x, \omega)-A_{d}(\omega)\right|^{2} d \omega \\
= & \frac{1}{2} x^{\prime} \Psi x+p^{\prime} x+W_{p} \omega_{p}
\end{aligned}
$$

where the weighting function $W(\omega)$ is given by

$$
W(\omega)= \begin{cases}W_{p} & \text { if } \omega \in \Omega_{p}, \\ W_{s} & \text { if } \omega \in \Omega_{s},\end{cases}
$$

and the positive definite matrix $\Psi$ (see [5] for details) and the column vector $p$ are, respectively, given by

$$
\begin{aligned}
& \Psi=2 W_{p} \int_{0}^{\omega_{p}} \xi(\omega) \xi^{\prime}(\omega) d \omega+2 W_{s} \int_{\omega_{s}}^{0.5} \phi(\omega) \xi^{\prime}(\omega) d \omega \\
& p=-2 W_{p} \int_{0}^{\omega_{p}} \xi(\omega) d \omega .
\end{aligned}
$$

Let $\delta_{p}$ and $\delta_{s}$ denote the maximum allowable passband and stopband errors, respectively. The PCWLSE filtering problem can be formulated as the following semi-infinite programming problem:

$$
\begin{aligned}
\min _{x} & \frac{1}{2} x^{\prime} \Psi x+p^{\prime} x \\
\text { s.t. } & \left|\xi^{\prime}(\omega) x-1\right| \leq \delta_{p}, \quad \forall \omega \in\left[0, \omega_{p}\right], \\
& \left|\xi^{\prime}(\omega) x\right| \leq \delta_{s}, \quad \forall \omega \in\left[\omega_{s}, 0.5\right] .
\end{aligned}
$$

This problem is transformed, by mapping the interval $\left[\omega_{s}, 0.5\right]$ onto $\left[0, \omega_{p}\right]$ and expanding the constraints into linear constraints, into the form of problem $(\mathrm{P})$ as follows,

$$
\begin{aligned}
& \min _{x} f(x)=\frac{1}{2} x^{\prime} \Psi x+p^{\prime} \\
& \text { s.t. } \quad A(\omega) x-c(\omega) \leq 0, \text { for all } \omega \in \Omega
\end{aligned}
$$

where

$$
A(\omega)=\left[\begin{array}{l}
\xi^{\prime}(\omega) \\
-\xi^{\prime}(\omega) \\
\xi^{\prime}\left(\omega_{s}+\frac{0.5-\omega_{s}}{\omega_{p}} \omega\right) \\
-\xi^{\prime}\left(\omega_{s}+\frac{0.5-\omega_{s}}{\omega_{p}} \omega\right)
\end{array}\right] \text { and } c(\omega)=\left[\begin{array}{l}
1+\delta_{p} \\
-1+\delta_{p} \\
\delta_{s} \\
\delta_{s}
\end{array}\right]
$$

and

$$
\Omega=\left[0, \omega_{p}\right]
$$


We assume that $\xi(\omega) \in C\left(\Omega, R^{n}\right)$ and the Slater constraint qualification is satisfied. Thus, Algorithm 2.1 is applicable for solving the PCWLSE filtering problem (3.7)(3.10). The corresponding parameterized dual problem is

$$
\begin{array}{ll}
\min _{(W, \lambda)} L_{k}(x, w, \lambda) \\
\text { s.t. } & \Psi x+P-\sum_{i=1}^{k} A\left(\omega_{i}\right)^{T} \lambda^{i}=0 \\
& 0 \leq \omega_{i} \leq \omega_{p}, \quad i=1,2, \cdots, k, \\
& \lambda_{i} \geq 0, i=1,2, \cdots, k,
\end{array}
$$

where $x \in R^{N}, w=\left[\omega_{1}, \ldots, \omega_{k}\right]^{\prime} \in R^{k}, \lambda=\left[\lambda^{1}, \lambda^{2}, \cdots, \lambda^{k}\right]$ with $\lambda^{i}=\left[\lambda_{1}^{i}, \lambda_{2}^{i}, \lambda_{3}^{i}, \lambda_{4}^{i}\right]$ for $i=1, \cdots, k$, and the cost function is given by

$$
L_{k}(x, w, \lambda)=\frac{1}{2} x^{\prime} \Psi x+\sum_{i=1}^{k} c^{\prime} \lambda^{i}
$$

where $c=\left[1+\delta_{p},-1+\delta_{p}, \delta_{s}, \delta_{s}\right]^{\prime}$.

Consider a lowpass FIR filter of length $N=35$ associated with parameters $\bar{\omega}_{p}=$ $0.05, \bar{\omega}_{s}=0.1, W_{p}=1$, and $W_{s}=1000$. Highpass, bandpass, and bandstop filters can be handled analogously. Let $D B_{p}=20 \log _{10}\left\{\left(1+\delta_{p}\right) /\left(1-\delta_{p}\right)\right\}$ and $D B_{s}=20 \log _{10} \delta_{s}$ represent, respectively, the passband and stopband amplitude ripples measured in decibels $(d B)$. The design objective requires $D B_{p}=1.0 d B$ and $D B_{s} \leq-40 d B$.

By using the algorithm of Section 2, problem (3.11)-(3.14) is solved. The numerical result is illustrated by Figures 1 and 2. Figure 1 shows comparison between the magnitude response of the initial filter $x_{1}$ and the magnitude response of the optimal filter $x_{\text {opt }}$ obtained from Step 3 of Algorithm 2.1. Clearly, the initial magnitude response $A\left(x_{1}, f\right)$ does not satisfy the passband and stopband constraints, while the optimal magnitude response $A\left(x_{o p t}, \omega\right)$ stays completely inside the constraints. Figure 2 shows the difference between the initial frequency response and the optimal frequency response. The straight dash-dot line depicted in Figure 2 denotes the constraint in the stopband, where the design of stopband attenuation is set to be less than or equal to $-40 \mathrm{~dB}$. The figure shows that the frequency response for the optimal filter meets all the constraints, while the initial frequency response does not. Comparison with other methods will be given at the end of Section 4 .

4. Time-domain Envelope-Constrained Filter Design. For the optimum envelope-constrained filter design problem considered in [3], [15] and [18], the design objective is to process a given input signal $s(t)$, which is corrupted by additive random noise $n(t)$, in such a way that the output noise enhancement is minimized, while the noise-free output $\Phi(t)$ is required to fit into a prescribed pulse-shaped envelope defined by lower and upper boundaries. The mathematical formulation of this optimum 
constrained filter design problem is:

$$
\begin{array}{ll}
\min _{u}\|u\|^{2} \\
\text { s.t. } & \varepsilon^{-}(t) \leq \Phi(t)^{\prime} \leq \varepsilon^{+}(t), \quad \forall t \in[0, \infty)
\end{array}
$$

where $u(t) \in L^{2}[0, \infty)$ is the impulse response of a time-invariant filter, $\varepsilon^{-}$and $\varepsilon^{+}$ are the lower and upper boundaries defining the envelope, and the noiseless output has support in $[0, \infty)$ and is expressed as:

$$
\Phi(t)=\int_{0}^{\infty} u(t) s(t-\tau) d \tau .
$$

If $\left\{\phi_{j}\right\}_{j=1}^{\infty}$ is an orthonormal basis of $L^{2}[0, \infty)$, then $u(t)$ can be expressed as:

$$
u(t)=\sum_{j=1}^{\infty} x_{j} \phi_{j}(t)
$$

and

$$
x_{j}=\left\langle u, \varphi_{j}\right\rangle, \quad j=1,2, \cdots .
$$

Under the orthonormal basis $\left\{\phi_{j}\right\}_{j=1}^{\infty}$, the $L^{2}$ norm of $u \in L^{2}(0, \infty)$ can be written as

$$
\|u\|=\sqrt{\sum_{j=1}^{\infty} x_{j}^{2}}
$$

We consider the problem in finite time and consider only those filters $u_{N}(t)$ whose impulse response is represented by finite expansion on the orthonormal basis:

$$
u_{N}(t)=\sum_{j=1}^{N-1} x_{j} \phi_{j}
$$

In this case, the output response can be expressed as

$$
\begin{aligned}
\Phi(t) & =\int_{0}^{T} u_{N}(t) s(t-\tau) d \tau \\
& =\Theta^{\prime}(t) x, \quad \forall t \in[0, T]
\end{aligned}
$$

where $x=\left[x_{0}, \cdots, x_{N-1}\right]^{\prime}, \Theta(t)=\left[\theta_{0}(t), \cdots, \theta_{N-1}(t)\right]^{\prime}$, and

$$
\theta_{j}(t)=\int_{0}^{T} \phi_{j}(\tau) s(t-\tau) d \tau, \quad j=0, \cdots, N-1 .
$$

The norm of $u_{N}$ becomes

$$
\left\|u_{N}\right\|=\sqrt{\sum_{i=0}^{N-1} \sum_{j=1}^{N-1} x_{i} x_{j}\left\langle\phi_{i}, \phi_{j}\right\rangle}=\sqrt{x^{\prime} x}
$$


where $\left\langle\phi_{i}, \phi_{j}\right\rangle=\delta_{i, j}$. Under the new settings, problem (4.1)-(4.2) is reduced to

$$
\begin{aligned}
& \min _{x}\|x\|^{2}=x^{\prime} x, \quad x \in R^{N} \\
& \text { s.t. } \varepsilon^{-}(t) \leq \Theta^{\prime}(t) x \leq \varepsilon^{+}(t), \quad \forall t \in[0, T] .
\end{aligned}
$$

Let

$$
A(t)=\left[\begin{array}{r}
\Theta(t)^{\prime} \\
-\Theta(t)^{\prime}
\end{array}\right],
$$

and

$$
b(t)=\left[\varepsilon^{+\prime}(t),-\varepsilon^{-\prime}(t)\right]^{\prime}
$$

The above EC filter problem can be written in the form of (2.1)-(2.2), where $\tau$ is replaced by $t, f(x)=\|x\|^{2}$ and $\Omega=[0, T]$. Now, as a specific numerical example, we consider the design of an equalization filter for a digital transmission channel consisting of a coaxial cable on which data is transmitted according to the DSX-3 standard [3]. The design objective is to find an equalizing filter to shape the impulse response of a coaxial cable with a loss of $30 \mathrm{~dB}$ at a normalized frequency of $1 / \beta$ as input, where $\beta$ is the baud interval $\left(22.35 \times 10^{-9} s\right)$. More precisely, an equalizing filter is to be obtained such that the objective function is minimized, while the output corresponding to a given input signal $(s(t), t \in[0, T])$ lies within the envelope given by the DSX-3 pulse template. The corresponding continuous-time EC filter design problem with $L^{2}([0, T])$ Laguerre orthonormal basis can be formulated in the following as a quadratic semi-infinite programming problem.

In this example, the interval $[0,32 \beta]$ is equally partitioned into $2^{10}$ subintervals. Then, the interval $[0,32 \beta]$ is represented by the $2^{10}+1$ end points of these subintervals. In this way, the analog input signal is sampled every $\beta / 32$ time unit. These sample signals suffice to give a good representation of the continuous signal. Furthermore, the output mask and the filter output are also discretized into $2^{10}$ points for the purpose of better display of the design results. However, we wish to note that the design technique proposed in this paper for obtaining the optimal solution does not require any discretization of the input signal, the output mask, or the filter output.

Algorithm 2.1 solves the problem efficiently. Figure 5 shows the comparison between the initial output response and the optimal output response obtained from Step 3 of Algorithm 2.1. Note that the optimal output response satisfies the required continuous envelope constraint at all times, while the initial output response violates the continuous envelope constraint.

We note that the design problems treated in this and the previous sections were solved, in [14] and [5] respectively, by a two stage optimization method. It first solves problem $(\mathrm{PD}(\mathrm{t}))$, for some chosen $t$, to get the optimal value function $v(t)$. Then the 
index vector $t$ is updated by optimization $v(t)$. The algorithm repeats the two steps until the solution is found. That algorithm suffers from a few disadvantages. First of all, the algorithm has no guaranteed convergence. The cost function $v(t)$ of the outer level optimization is not even smooth. In contrast, the new algorithm is supported by a guaranteed convergence result.

5. Summary and Conclusion. This paper gave a unified quadratic semiinfinite programming approach to the design of optimal filters subject to either time or frequency domain specifications. The algorithm so obtained is guaranteed to find an optimal solution satisfying the continuous constraints of the original problem if it exists. The proposed design technique has an advantage over existing methods in terms of computational efficiency and theoretical completeness.

\section{REFERENCES}

[1] J. W. AdAms, FIR digital filters with least-square stopbands subject to peak-gain constraints, IEEE Trans. Circuits Syst., 39(1991), pp. 376-388.

[2] J. W. Adams and J. L. Sullivan, Peak-constrained least square optimization, IEEE Trans. Signal Processing, 46(1998), pp. 306-321.

[3] Bell Communications, DSX-3 isolated pulse template and equations, Tech. Ref. TR-TSY000499, Iss. 2, pp. 9-17, Dec. 1988.

[4] Physical/electrical characteristic of hierarchical digital interfaces, G.703, Fascicle III, 1991.

[5] H. H. DAm, K. L. TeO, S. NoRdebo, And A. CANToni, The dual parameterization approach to optimal least square FIR filter design subject to maximum error constraints, IEEE Trans. Signal Processing, 48:8(2000), pp. 2314-2320.

[6] M. J. Grimble And A. E. SAyed, Solution of the $H^{\infty}$ optimal linear filtering problem for discrete-time systems, IEEE Trans. Acoustic, Speech, and signal processing, 38(1990), pp. 1092-1104.

[7] S. Ito, Y. Liu, K. L. Teo, A dual parametrization method for convex semi-infinite programming, Annals of Operations Research, 98(2000), pp. 189-213.

[8] Y. Liu, K. L. Teо, And S. Iто, Global optimization in quadratic semi-infinite programming, to appear in Computing.

[9] T. W. Parks and C. S. Burrus, Digital Filter Design, New York: Wiley, 1987.

[10] Z. TAN, Y. C. Sho, AND L. XIE, $H_{2}$ optimal envelope-constrained FIR filter design: an LMI approach, Signal Processing, 75(1999), pp. 141-149.

[11] Z. TAn, Y. C. Sho, And L. XIE, Envelope-constrained $H_{\infty}$ FIR filter design, IEEE Trans. Circuis and Systems II, 47(2000), pp. 79-82.

[12] K. L. Teo, C. J. Goh, and K. H. Wong, A Unified Computational Approach to Optimal Control Problems, Longman Scientific and Technical, Essex, England, 1991.

[13] K. L. Teo, V. Rehbock, And L. S. Jennings, A New Computational Algorithm for Functional Inequality Constrained Optimization Problems, Automatica, 29(1993), pp. 789-792.

[14] C. H. Tseng, Iterative Algorithms for Envelope-Constrained Filter Design, Ph.D thesis, Curtin University of Technology, 1999.

[15] C. H. Tseng, K. L. Teo, A. Cantoni, and Z. Zang, A dual approach to continuous-time envelope-constrained filter design via orthonormal filters, IEEE Trans. Circuits Syst.-I: Fundamental Theory and Applications, 46:9(1999), pp. 1042-1054. 
[16] B. Vo, A. CANToni, AND K. L. TEO, Envelope-constraned filter with linear interpolator, IEEE Trans. Signal Processing, 45(1997), pp. 1405-1414.

[17] Z. Zang, A. Cantoni, And K. L. TeO, Envelope-constrained FIR filter design via $H_{\infty}$ optimization methods, IEEE Trans. Circuis and Systems II, 46(1999), pp. 649-653.

[18] B. Vo, Z. ZAng, A. Cantoni And K. L. Teo, Continuous-time envelope-constrained filter design via orthonormal filters, IEE Proc.-Vis. Image Signal Process., 142:6(1995), pp. 389-394.

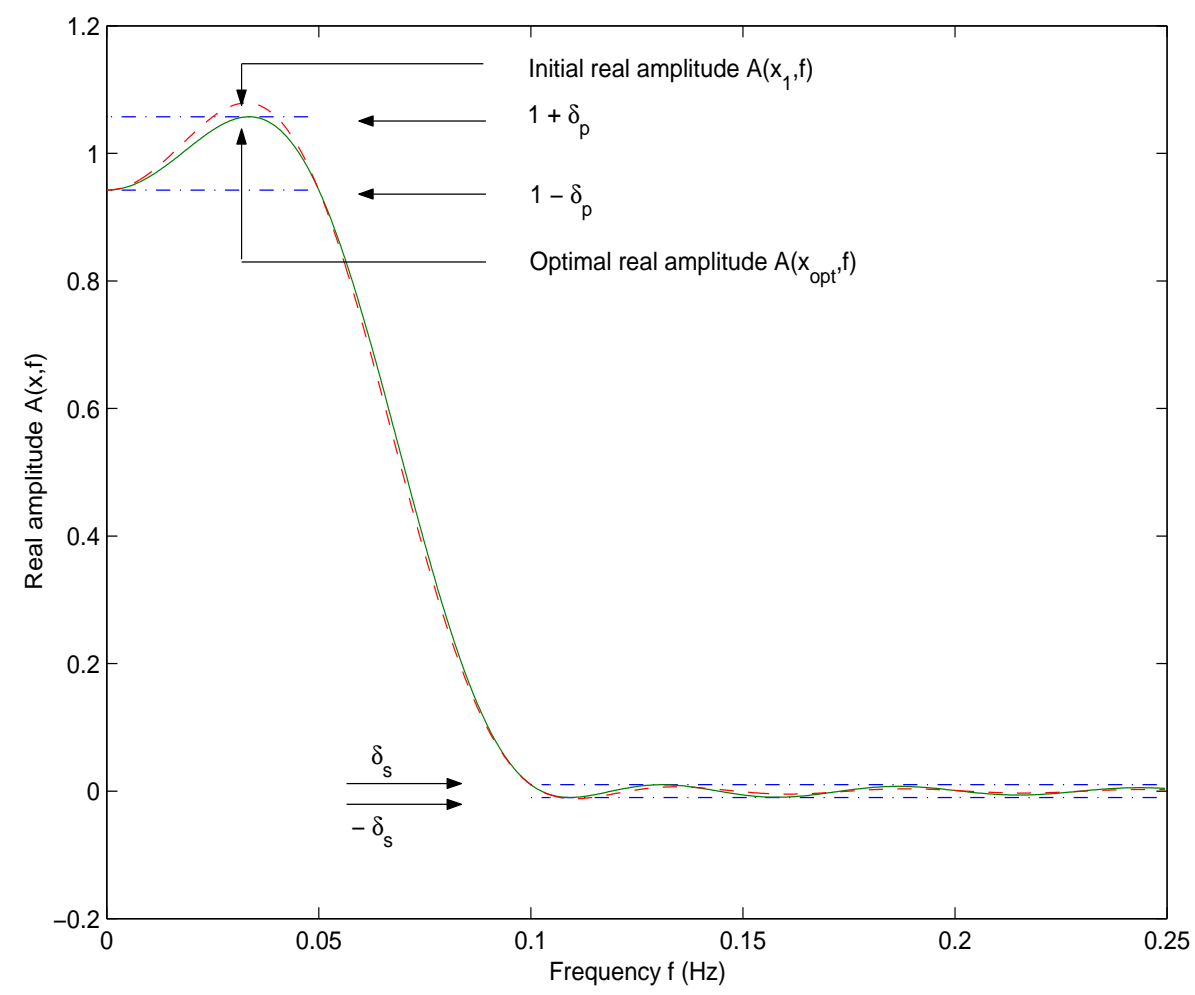

FIG. 1. Magnitude response of initial and optimal filters 


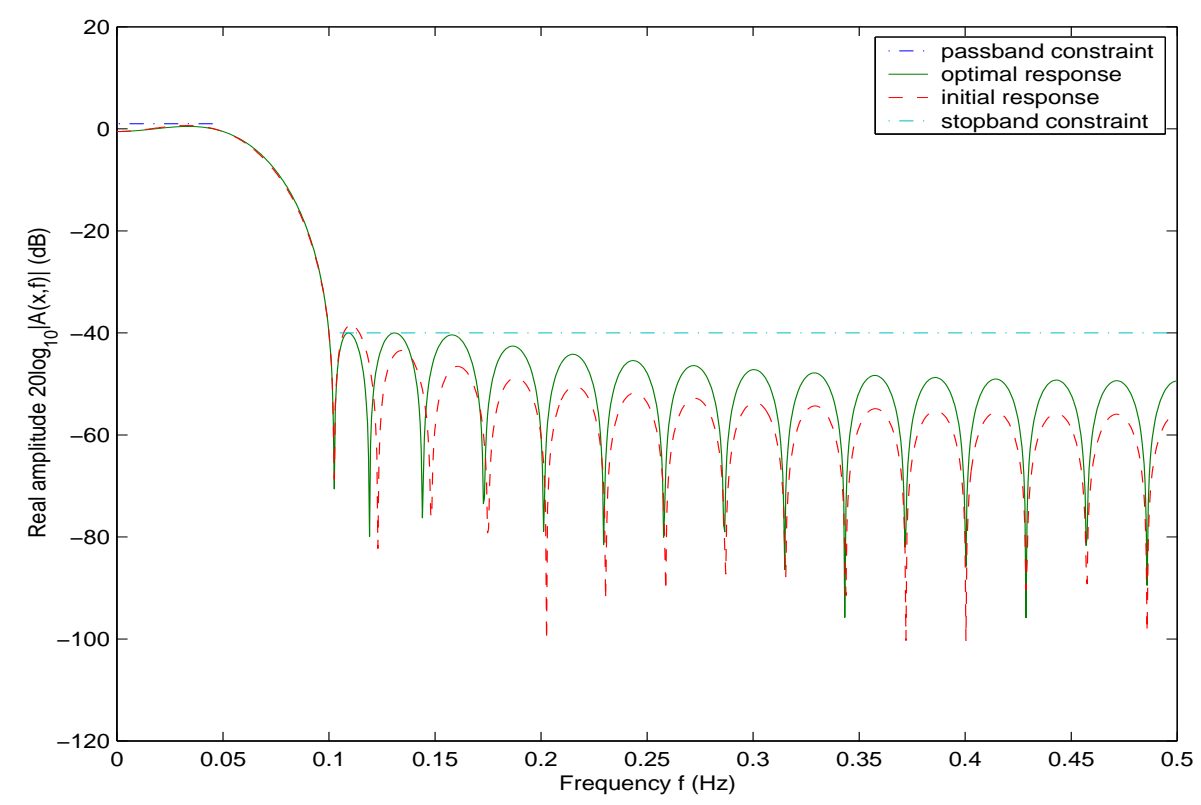

FIG. 2. Frequency response of initial and optimal filters

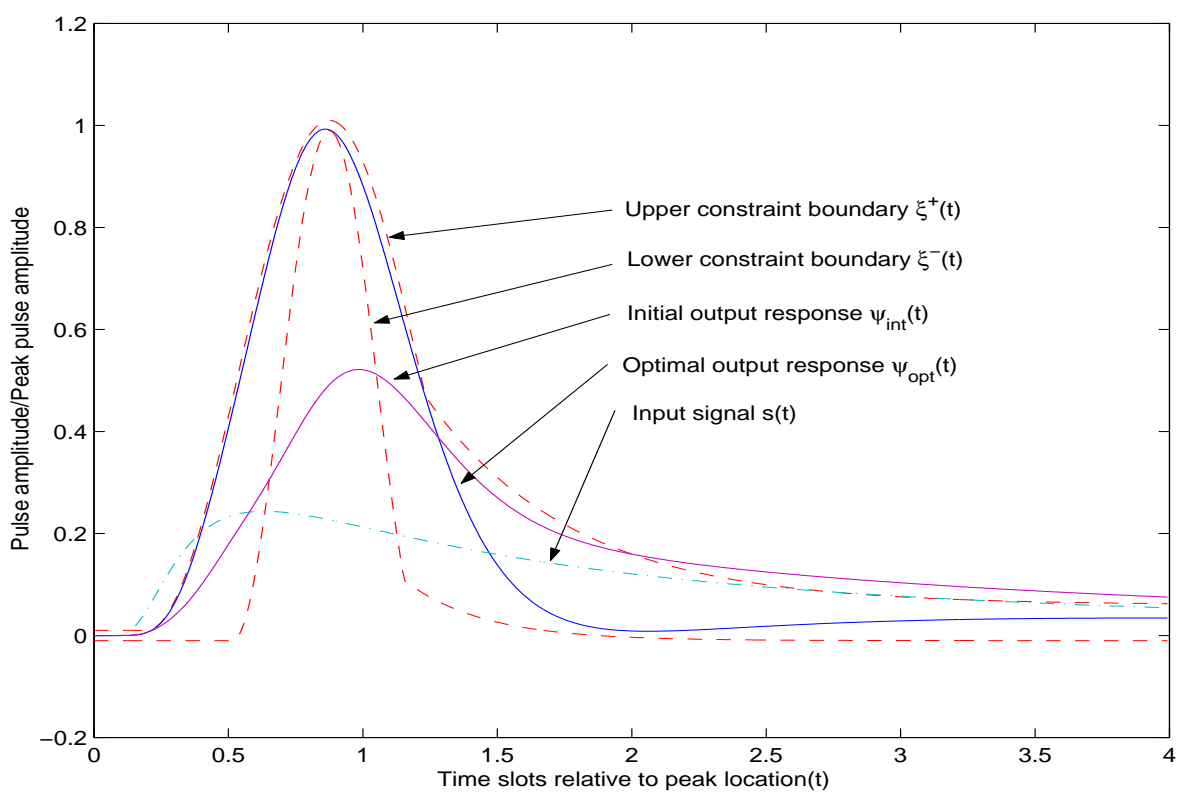

FIG. 3. Comparison between the optimal output response and initial output response in the DSX-3 pulse template coaxial cable. 